\title{
Optimal binning of X-ray spectra and response matrix design
}

\author{
J. S. Kaastra ${ }^{1,2,3}$ and J. A. M. Bleeker ${ }^{1,3}$ \\ 1 SRON Netherlands Institute for Space Research, Sorbonnelaan 2, 3584 CA Utrecht, The Netherlands \\ e-mail: J.S.Kaastra@sron.nl \\ 2 Leiden Observatory, Leiden University, PO Box 9513, 2300 RA Leiden, The Netherlands \\ 3 Department of Physics and Astronomy, Universiteit Utrecht, PO Box 80000, 3508 TA Utrecht, The Netherlands
}

Received 18 September 2015 / Accepted 8 January 2016

\begin{abstract}
Aims. A theoretical framework is developed to estimate the optimal binning of X-ray spectra.

Methods. We derived expressions for the optimal bin size for model spectra as well as for observed data using different levels of sophistication.

Results. It is shown that by taking into account both the number of photons in a given spectral model bin and their average energy over the bin size, the number of model energy bins and the size of the response matrix can be reduced by a factor of 10-100. The response matrix should then contain the response at the bin centre as well as its derivative with respect to the incoming photon energy. We provide practical guidelines for how to construct optimal energy grids as well as how to structure the response matrix. A few examples are presented to illustrate the present methods.
\end{abstract}

Key words. instrumentation: spectrographs - methods: data analysis - X-rays: general

\section{Introduction}

Until two decades ago X-ray spectra of cosmic X-ray sources were obtained using instruments such as proportional counters and gas scintillation proportional counters with moderate spectral resolution (typically 5-20\%). With the introduction of charge-coupled devices (CCDs) (ASCA, launch 1993) a major leap in energy resolution has been achieved (up to 2\%) and very high resolution has become available through grating spectrometers, first on the Extreme Ultraviolet Explorer (EUVE), and later on the Chandra and XMM-Newton observatories.

These X-ray spectra are usually analysed with a forwards folding technique. First a spectral model appropriate for the observed source is chosen. This model is convolved with the instrument response, which is represented usually by a response matrix. The convolved spectrum is compared to the observed spectrum and the parameters of the model are varied in a fitting procedure in order to obtain the best solution.

This classical way of analysing X-ray spectra has been widely adopted and is implemented, e.g. in spectral fitting packages such as XSPEC (Arnaud 1996), SHERPA (Freeman et al. 2001), and SPEX (Kaastra et al. 1996). However, the application of standard concepts, such as a response matrix, is not at all trivial for high-resolution instruments. For example, with the Reflection Grating Spectrometer (RGS) of XMM-Newton, the properly binned response matrix is 120 Megabytes in size, counting only non-zero elements. Taking into account that usually data from both RGS detectors and of two spectral orders are fit simultaneously, makes it at best slow to handle even by most present day computer systems. Also the higher spectral resolution considerably enhances the computation time needed to evaluate the spectral models. Since the models applied to Chandra and XMM-Newton data are much more complex than those applied to data from previous missions, computational efficiency is important to take into consideration.
For these reasons we discuss here the optimal binning of both model spectra and data. In this paper, we critically re-evaluate the concept of response matrices and the way spectra are analysed. In fact, we conclude that it is necessary to drop the classical concept of a matrix, and to use a modified approach instead.

The outline of this paper is as follows. We start with a more in-depth discussion regarding the motivation for this work (Sect. 2). In the following sections, we discuss the classical approach to spectral modelling and its limitations (Sect. 3), the optimal bin size for model spectra (Sect. 4) and data (Sect. 5) followed by a practical example (Sect. 6). We then turn to the response matrix (Sect. 7), its practical construction (Sect. 8), and briefly to the proposed file format (Sect. 9) before reaching our conclusions.

\section{Motivation for this work}

In this section we present in more depth the arguments leading to the proposed binning of model spectra and observational data and our choice for the response matrix design. We do this by addressing the following questions.

\subsection{Why not use straightforward deconvolution?}

In high-resolution optical spectra the instrumental broadening is often small compared to the intrinsic line widths. In those cases it is common practice to obtain the source spectrum by dividing the observed spectrum at each energy by the nominal effective area (straightforward deconvolution).

Although straightforward deconvolution, due to its simplicity would seem to be attractive for high-resolution X-ray spectroscopy, it fails in several situations. For example, spectral orders may overlap as with the EUVE spectrometers (Welsh et al. 1990) or the Chandra (Weisskopf et al. 1996) Low-Energy 
Transmission Grating Spectrometer (LETGS; Brinkman et al. 2000) when the HRC-S detector is used. In these cases only careful instrument calibration in combination with proper modelling of the short wavelength spectrum can help. In case of the RGS (Den Herder et al. 2001) on board XMM-Newton (Jansen et al. 2001) $30 \%$ of all line flux is contained in broad wings as a result of scattering on the mirror and gratings, and this flux is practically impossible to recover by straightforward deconvolution. Finally, high-resolution X-ray spectra often suffer from both severe line blending and relatively large statistical errors because of low numbers of counts in some parts of the spectrum. This renders the method unsuitable.

\subsection{Why are high-resolution spectra much more complex to handle than low-resolution spectra?}

The enhanced spectral resolution and sensitivity of the current high-resolution X-ray spectrometers require much more complex source models with a multitude of free parameters as compared to the crude models that suffice for low-resolution spectra to cover all relevant spectral details that can be exposed by the far superior resolving power of state-of-the-art cosmic X-ray spectrometers. This does not merely involve adding more lines to the old models with the same number of parameters. Whereas investigators first fit single-temperature, solar abundance spectra, now multi-temperature, free abundance models are to be employed for stars and clusters of galaxies, among others. Models of AGN have evolved from simple power laws with a Gaussian line profile occasionally superimposed to complex continua spectra, including features due to reflection, relativistic blurring, soft excesses, multiple absorbing photo-ionised outflow components, low-ionisation emission contributions originating at large distances, which yet again leads to much larger numbers of free parameters to be accounted for.

\subsection{Why is it not possible to model complex spectra by the sum of a simple continuum plus delta lines?}

Practically all X-ray spectra in the Universe are too complex to simulate with simple continuum shapes with superimposed $\delta$-functions mimicking line features when observed at high resolution. Apart from spectral lines, other narrow features often found are: for example, radiative recombination continua, absorption edges, Compton shoulders, or dust features. Moreover spectral lines are too complex to be dealt with by delta functions, i.e. the astrophysics deriving from Doppler broadening or natural broadening, or a combination thereof (Voigt profiles) remains totally unaccounted for.

Even if one would model a line emission spectrum by the sum of $\delta$-lines, however, a physical model connecting the line intensities is needed. The line fluxes are mutually not independent and, as a consequence, many weak lines may not be detected individually but added together they may give a detectable signal.

Furthermore, except for the nearest stars, almost all X-ray sources are subject to foreground interstellar absorption, also yielding narrow and sharp spectral features such as absorption edges or absorption lines (for instance the well-known O I 1s-2p transition at $23.5 \AA$ ).

As an example, Seyfert 1 galaxies show a range of emission lines with different widths, often superimposed on each other and corresponding to the same transition, from narrow-line region lines (width few $100 \mathrm{~km} \mathrm{~s}^{-1}$ ), intermediate width lines $\left(1000 \mathrm{~km} \mathrm{~s}^{-1}\right.$ ), broad-line region lines (several 1000 up to tens of thousands $\mathrm{km} \mathrm{s}^{-1}$ ) up to relativistically broadened lines. In such cases splitting in narrow- and broadband features is impossible, as there is spectral structure at many different (Doppler) scales. As already stated above, there is a need for physically relevant models, regrettably, $\delta$-functions do not qualify for this.

In principle, the user could design an input model energy grid where there is only substantially higher spectral resolution at the energies where the model would predict narrow spectral features, for instance near foreground absorption edges or strong sharp emission lines. However, the real source spectrum may contain additional narrow features that are not anticipated by the user, and we want to avoid recreating grids and matrices multiple times. For this reason, the binning scheme proposed in this paper only depends on the properties of the instrument and the observed spectrum in terms of counts per resolution element.

\subsection{Why not use precalculated model grids?}

Some spectral models are computationally intensive. We are aware of the method of precalculating grids of models, such that the spectral fitting proceeds faster. This is common practice with for instance the APEC model for collisional plasmas as implemented in XSPEC or XSTAR models for photo-ionised plasmas. For a limited set of parameters this is an acceptable solution. However, as has been outlined above in many situations, astrophysical models require substantially more free parameters than can be provided with grids for 2-4 free parameters.

As an example, for stellar spectra not only temperature but also density and the UV radiation field (for He-like triplets) constitute important parameters. However, the standard APEC implementation only has temperature and abundances as free parameters.

Photo-ionised, warm absorber models for AGN depend nonlinearly on the shape of the ionising spectral energy distribution and on source parameters such as abundances and turbulence. In realistic descriptions, this requires at least five or more free parameters and is much too expensive to construct grids. Using grids in such cases always implies less realistic models.

In addition, creating these grids offers efficiency gain in the fitting procedure but shifts the computational burden to the grid creation. The developers of APEC communicated to us that a full grid covering all relevant temperatures requires of the order of a week computation time. A single XSTAR model (1 spectrum) takes on average about 20 min to complete, hence even modest grids may take a week or so to complete. So, also it is extremely useful to be able to limit the number of energy bins in those cases.

\subsection{Why not use brute force computing power?}

We note that for individual spectra extensive energy grids and large sizes of response matrices can be handled with presentday computers, but the computation time in folding the response matrix into the spectrum is substantial. This holds, in particular, if this process has to be repeated thousands of times in spectral fitting and error searches for models with many free parameters.

More importantly, in several cases users want to fit spectra of time-variable sources taken at different epochs together, using spectral models where some parameters are fixed between the observations while others are not. This requires loading as many response matrices as number of observations, and we have seen cases where such analyses simply cannot be carried out in this way. 
There are also other cases where investigators have to rely on indirect methods such as making maps of line centroids or equivalent widths, rather than full spectral fitting. A striking example constitutes the Chandra CCD spectra of Cas A. With 1 arcsec spatial resolution the remnant covers of order $10^{5}$ independent regions, which should ideally be fit together with a common spectral model with position-dependent parameters.

\subsection{Some explicit examples of spectral analyses that require considerable computation time}

A few typical examples may help to stipulate the relevance of our case regarding required computing time.

Mernier et al. (2015) analysed a cluster of galaxies with the XMM-Newton EPIC detectors. These detectors still have a modest spectral resolution. The spectra of three detectors in two observations with different background levels were fit jointly. The model was a multi-temperature plasma model with about 50 free parameters, including abundances and different instrumental and astrophysical background components. Fits including error searches took about $5-10 \mathrm{~h}$ on a modern workstation. Additional time is needed to verify that fits do not get stuck in local subminima. These authors are extending the work now to radial profiles in clusters, incorporating eight annuli per cluster, on a sample of 40 clusters. Several months of cpu time using multiple workstations are needed to complete this analysis.

Kaastra et al. (2014a) studied the AGN Mrk 509. Chandra HETGS spectra were fit jointly with archival XMM-Newton spectra. As a result of the amount of information available by virtue of the high spectral resolution, the spectrum was modelled using an absorption model that comprised the product of 28 components (four velocity components times seven ionisation components). With the inclusion of free parameters for the modelling of the continuum and the narrow and broad emission features, the model ended up with about 60 free parameters. It took two weeks of computing time to obtain the final model including the assessment of uncertainties on all parameters.

Kaastra et al. (2014b) observed the Seyfert galaxy NGC 5548 in an obscured state. Stacked RGS, pn, NuSTAR, and INTEGRAL data were analysed jointly. The model (table S3 of that paper) had 35 free parameters and consisted of six "warm absorber" components and two "obscured" components, giving a total of eight multiplied transmission factors, which had to be determined iteratively in about eight steps to reach full convergence. This is because the inner, obscurer components affect the ionising spectral energy distribution for the outer, warm absorber components. At each intermediate step a full grid of Cloudy models had to be calculated to update the transmittance of the obscured nuclear spectrum. The full computation also took several days in addition to months of trial to set up the proper model and calculation scheme.

\section{Classical approach to spectral modelling and its limitations}

\subsection{Response matrices}

The spectrum of an X-ray source is given by its photon spectrum $f(E)$, a function of the continuous variable $E$, the photon energy, and has units of, e.g. photons $\mathrm{m}^{-2} \mathrm{~s}^{-1} \mathrm{keV}^{-1}$. To produce the predicted count spectrum $s\left(E^{\prime}\right)$ measured by an instrument, $f(E)$ is convolved with the instrument response $R\left(E^{\prime}, E\right)$ as follows:

$$
s\left(E^{\prime}\right)=\int_{0}^{\infty} R\left(E^{\prime}, E\right) f(E) \mathrm{d} E,
$$

where the data channel $E^{\prime}$ denotes the observed (see below) photon energy and $s$ has units of counts $\mathrm{s}^{-1} \mathrm{keV}^{-1}$. The response function $R\left(E^{\prime}, E\right)$ has the dimensions of an (effective) area, and can be given in, e.g. $\mathrm{m}^{2}$.

The variable $E^{\prime}$ is denoted as the observed photon energy, but in practice it is often some electronic signal in the detector, the strength of which usually cannot take arbitrary values, but can have only a limited set of discrete values, for instance as a result of analogue to digital conversion (ADC) in the processing electronics. A good example of this is the pulse-height channel for a CCD detector. Alternatively, it may be the pixel number on an imaging detector if gratings are used.

In almost all circumstances it is not possible to carry out the integration in Eq. (1) analytically because of the complexity of both the model spectrum and instrument response. For that reason, the model spectrum is evaluated at a limited set of energies, corresponding to the same predefined set of energies that is used for the instrument response $R\left(E^{\prime}, E\right)$. Then the integration in Eq. (1) is replaced by a summation. We call this limited set of energies the model energy grid or for short the model grid. For each bin $j$ of this model grid, we define a lower and upper bin boundary $E_{1 j}$ and $E_{2 j}$, a bin centre $E_{j}=0.5\left(E_{1 j}+E_{2 j}\right)$, and a bin width $\Delta E_{j}=E_{2 j}-E_{1 j}$.

Provided that the bin width of the model energy bins is sufficiently small compared to the spectral resolution of the instrument, the summation approximation to (1) is in general accurate. The response function $R\left(E^{\prime}, E\right)$ has therefore been replaced by a response matrix $R_{i j}$, where the first index $i$ denotes the data channel, and the second index $j$ the model energy bin number.

We have explicitly

$$
S_{i}=\sum_{j} R_{i j} F_{j}
$$

where now $S_{i}$ is the observed count rate in counts s${ }^{-1}$ for data channel $i$ and $F_{j}$ is the model spectrum (photons $\mathrm{m}^{-2} \mathrm{~s}^{-1}$ ) for model energy bin $j$.

\subsection{Evaluation of the model spectrum}

The model spectrum $F_{j}$ can be evaluated in two ways. First, the model can be evaluated at the bin centre $E_{j}$, essentially taking

$F_{j}=f\left(E_{j}\right) \Delta E_{j}$.

This is appropriate for smooth continuum models such as blackbody radiation and power laws. For line-like emission, it is more appropriate to integrate the line flux within the bin analytically, taking

$F_{j}=\int_{E_{1 j}}^{E_{2 j}} f(E) \mathrm{d} E$.

Now a serious flaw occurs in most spectral analysis codes. The parameter $S_{i}$ is evaluated in a straightforward way using (2). Hereby it is tacitly assumed that all photons in the model bin 
$j$ have exactly the energy $E_{j}$. This is necessary since all information on the energy distribution within the bin is lost and $F_{j}$ is essentially only the total number of photons in the bin. If the model bin width $\Delta E_{j}$ is sufficiently small this is no problem, however, this is (most often) not the case.

An example of this is the standard response matrix for the ASCA SIS detector (Tanaka et al. 1994), in which the investigators used a uniform model grid with a bin size of $10 \mathrm{eV}$. At a photon energy of $1 \mathrm{keV}$, the spectral resolution (full width at half maximum; FWHM) of the instrument was about $50 \mathrm{eV}$, hence the line centroid of an isolated narrow line feature containing $N$ counts can be determined with a statistical uncertainty of $50 /(2.35 \sqrt{N}) \mathrm{eV}$. We assume here for simplicity a Gaussian instrument response (FWHM is approximately $2.35 \sigma$, see Eq. (6)). Thus, for a line with 400 counts the line centroid can be determined with an accuracy of $1 \mathrm{eV}$, ten times better than the bin size of the model grid. If the true line centroid is close to the boundary of the energy bin, there is a mismatch (shift) of $5 \mathrm{eV}$ between the observed count spectrum and the predicted count spectrum at about the $5 \sigma$ significance level. If there are more of these lines in the spectrum, it is possible that a satisfactory fit (e.g. acceptable $\chi^{2}$ value) is never obtained, even in cases where the true source spectrum is known and the instrument is perfectly calibrated. The problem becomes even more worrisome if, for example detailed line centroiding is performed to derive velocity fields.

A simple way to resolve these problems is just to increase the number of model bins. This robust method always works, but at the expense of a lot of computing time. For CCD-resolution spectra this is perhaps not a problem, but with the increased spectral resolution and sensitivity of the grating spectrometers of Chandra and XMM-Newton this becomes cumbersome.

For example, the LETGS spectrometer of Chandra (Brinkman et al. 2000) has a spectral resolution (FWHM) between $0.040-0.076 \AA$ over the $1-175 \AA$ band. A 85 ks observation of Capella (obsid. 1248, Mewe et al. 2001; Ness et al. 2001) produced $N=14000$ counts in the Fe XVII line at $15 \AA$. Because line centroids can be determined with a statistical accuracy of $\sigma / \sqrt{N}$, this makes it necessary to have a model energy grid bin size $\sigma / \sqrt{N}$ of about $0.00014 \AA$, corresponding to 278 bins per FWHM resolution element and requiring 1.2 million model bins up to $175 \AA$ for a uniform wavelength grid. Although this small bin size is less than the (thermal) width of the line, larger model bin sizes would lead to a significant shift of the observed line with respect to the predicted line profile and a corresponding significant worsening of the goodness of fit. With future, more sensitive instruments like Athena (Nandra et al. 2013) such concerns will become even more frequent.

Most of the computing time in thermal plasma models stems from the evaluation of the continuum. The radiative recombination continuum has to be evaluated for all energies, for all relevant ions and for all relevant electronic subshells of each ion. On the other hand, the line power needs to be evaluated only once for each line, regardless of the number of energy bins. Therefore the computing time is approximately proportional to the number of bins. Therefore, a factor of 1000 increase in computing time is implied from the used number of ASCA-SIS bins (1180) to the required number of bins for the LETGS Capella spectrum (1.2 million bins).

Furthermore, because of the higher spectral resolution of grating spectrometers compared with CCD detectors, more complex spectral models are needed to explain the observed spectra, with more free parameters, which also leads to additional computing time. Finally, the response matrices of instruments like the XMM-Newton RGS become extremely large owing to extended scattering wings caused by the gratings.

It is therefore important to keep the number of energy bins as small as possible while maintaining the required accuracy for proper line centroiding. Fortunately there is a more sophisticated way to evaluate the spectrum and convolve it with the instrument response. Basically, if more information on the distribution of photons within a bin is taken into account (like their average energy), energy grids with broader bins (and hence with substantially fewer bins) can give results as accurate as fine grids where all photons are assumed to be at the bin center.

\section{What is the optimal bin size for model spectra?}

\subsection{Definition of the problem}

In the example of the LETGS spectrum of Capella we showed that to maintain full accuracy for the strong and narrow emission lines, very small bin sizes are required, which leads to more than a million grid points for the model spectrum.

Fortunately, there are several ways to improve the situation. Firstly, the spectral resolution of most instruments is not constant, and one might adapt the binning to the local resolution. Some X-ray instruments use this procedure. It helps, but the improvement is not very good for instruments like LETGS with a difference of only a factor of two in resolution from short to long wavelengths.

Secondly, the finest binning is needed near features with large numbers of counts, for instance the strong spectral lines of Capella. One might therefore adjust the binning according to the properties of the spectrum: narrow bins near high-count regions and broader bins near low-count regions. As far as we know, no X-ray mission utilises this procedure. Perhaps the main reason is that one first needs to know the observed count spectrum before being able to create the model energy grid for the response matrix. In most cases, the redistribution matrix is either precalculated for all spectra, or is calculated on the fly for individual spectra only to account for time-dependent instrumental features.

A third way to reduce the number of bins is to revisit the way model spectra are calculated. As indicated before, in most standard procedures, the integrated number of photons in a bin is calculated. In the convolution with the response matrix it is then tacitly assumed that all photons of the bin have the same energy, i.e. the energy $E_{j}$ of the bin centre. However, it could well be that the bin contains only one spectral line; it makes a difference if the line is at the lower or upper limit of the energy bin or at the bin centre. The proper centroid of the line in the observed count spectrum, after convolution with the response matrix, is only reproduced if in the model spectrum not only the number of photons but also their average energy is accounted for. As we show (see Fig. 1), accounting for the average energy of the photons within a bin allows us to have an order of magnitude larger bin sizes.

In the procedure proposed here we combine all three options to reduce the number of model energy bins: making use of the local spectral resolution, the strength of the spectral features in number of photons $N$, and accounting for the average energies of the photons within bins. 


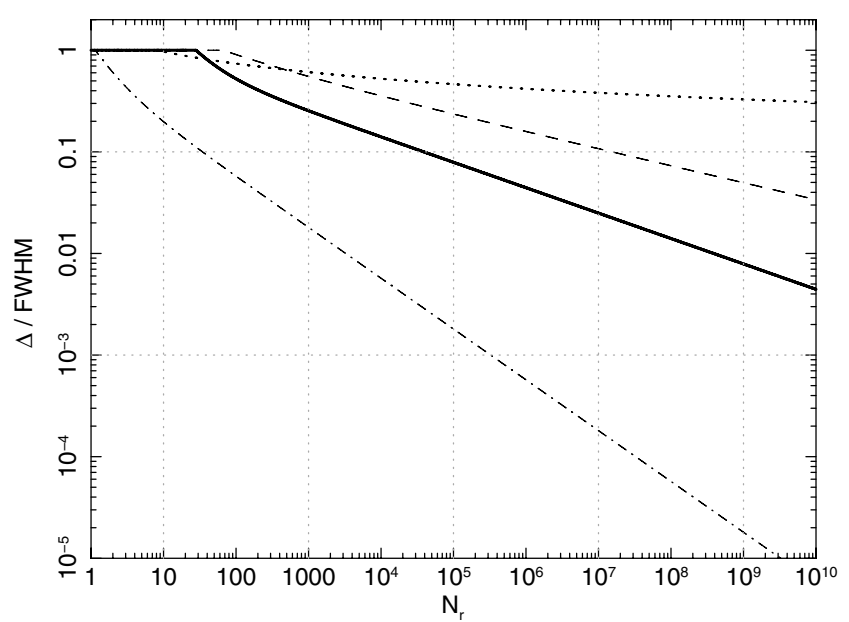

Fig. 1. Optimal bin size $\Delta$ for model spectrum binning with a Gaussian lsf, as a function of the number of counts per resolution element $N_{\mathrm{r}}$. Dash-dotted line: zeroth order approximation (18); thick solid line: first order approximation (19); dashed line: second order approximation (20). For comparison we also show as the dotted curve the approximation (36) for the data grid binning. All results shown are for $R=1$.

\subsection{Binning the model spectrum}

\subsubsection{Zeroth order approximation}

We have seen in the previous subsection that the classical way to evaluate model spectra is to calculate the total number of photons in each model energy bin, and to act as if all flux is at the centre of the bin. In practice, this implies that the true spectrum $f_{0}(E)$ within the bin is replaced by its zeroth order approximation, $f_{1,0}(E)$, and is written as

$f_{1,0}(E)=N \delta\left(E-E_{j}\right)$,

where $N$ is the total number of photons in the bin, $E_{j}$ the bin centre as defined in the previous section, and $\delta$ is the Dirac delta-function.

We assume, for simplicity, that the instrument response is Gaussian, centred at the true photon energy and with a standard deviation $\sigma$. Many instruments have line spread functions (lsf) with Gaussian cores. For instrument responses with extended wings (e.g. a Lorentz profile) the model binning is a less important problem, since in the wings all spectral details are washed out, and only the lsf core is important. For a Gaussian profile, the FWHM of the lsf is written as

$F W H M=\sqrt{\ln (256)} \sigma \simeq 2.35 \sigma$.

How can we measure the error introduced with approximation (5)? We compare the cumulative probability distribution functions (cdf) of the true spectrum and the approximation (5), which are both convolved with the instrumental lsf. The approach is described in detail in Sect. A, using a KolmogorovSmirnov test we derive the model bin size $\Delta E$ for which in $97.5 \%$ of all cases the approximation (5) leads to the same conclusion as a test using the exact distribution $f_{0}$ in tests of $f_{0}$ versus any alternative model $f_{2}$.

The approximation Eq. (5) fails most seriously in the case that the true spectrum within the bin is also a $\delta$-function, but located at the bin boundary, at a distance $\Delta / 2$ from the assumed line position at the bin centre.

The maximum deviation $\delta_{k}$ of the absolute difference of both cumulative distribution functions, $\delta_{k}=\left|F_{0}(x)-F_{1,0}(x)\right|$ (see also
Eq. (A.3)) occurs where $f_{0}(x)=f_{1,0}(x)$, as outlined at the end of Sect. A.3. Because $f_{1,0}(x)=f_{0}(x-\Delta / 2)$, we find that the maximum occurs at $x=\Delta / 4$. After some algebra we find that in this case

$\delta_{k}=\delta_{k, 0}=P(\Delta / 4)-P(-\Delta / 4)=2 P(\Delta / 4)-1=\frac{\Delta}{2 \sqrt{2 \pi} \sigma}$,

where $P$ is the cumulative normal distribution. This approximation holds in the limit of $\Delta \ll \sigma$. Inserting (6) we find that the bin size should be smaller than

$\frac{\Delta}{F W H M}<2.1289 \lambda_{k} N^{-0.5}$

where the number $\lambda_{k}$ is defined by (A.9). Monte Carlo results are presented in Sect. 4.3.

\subsubsection{First order approximation}

A further refinement can be reached as follows. Instead of putting all photons at the bin centre, we can put them at their average energy. This first-order approximation can be written as

$f_{1,1}(E)=N \delta\left(E-E_{\mathrm{a}}\right)$,

where $E_{\mathrm{a}}$ is given by

$E_{\mathrm{a}}=\int_{E_{1 j}}^{E_{2 j}} f(E) E \mathrm{~d} E$.

For the worst case zeroth order approximation $f_{1,0}$ for $f_{0}$, namely the case that $f_{0}$ is a narrow line at the bin boundary, the approximation $f_{1,1}$ yields exact results. Thus, it constitutes a major improvement. In fact, it is easy to see that the worst case for $f_{1,1}$ is a situation where $f_{0}$ consists of two $\delta$-lines of equal strength: one at each bin boundary. In that case, the width of the resulting count spectrum is broader than $\sigma$. Again in the limit of small $\Delta$, it is easy to show that the maximum error $\delta_{k, 1}$ for $f_{1,1}$ to be used in the Kolmogorov-Smirnov test is written as

$\delta_{k, 1}=\frac{1}{8 \sqrt{2 \pi e}}\left(\frac{\Delta}{\sigma}\right)^{2}$,

where $e$ is the base of the natural logarithms. Accordingly, the limiting bin size for $f_{1,1}$ is expressed as

$\frac{\Delta_{1}}{F W H M}<2.4418 \lambda(R)^{0.5} N^{-0.25}$.

It is seen immediately that for large $N$ the approximation $f_{1,1}$ requires a significantly smaller number of bins than $f_{1,0}$.

\subsubsection{Second order approximation}

We can decrease the number of bins further by not only calculating the average energy of the photons in the bin (the first moment of the photon distribution), but also its variance (the second moment). In this case we approximate

$\left.f_{1,2}(E)=N \exp \left[\left(E-E_{\mathrm{a}}\right) / 2 \tau^{2}\right)\right]$, 
where $\tau$ is given by

$$
\tau^{2}=\int_{E_{1 j}}^{E_{2 j}} f(E)\left(E-E_{\mathrm{a}}\right)^{2} \mathrm{~d} E .
$$

The resulting count spectrum is then simply Gaussian with the average value centred at $E_{\mathrm{a}}$ and the width slightly larger than the instrumental width $\sigma$, namely $\sqrt{\sigma^{2}+\tau^{2}}$.

The worst case again occurs for two $\delta$-lines at the opposite bin boundaries, but now with unequal strength. It can be shown in the small bin width limit that

$$
\delta_{1,2}=\frac{1}{36 \sqrt{6 \pi}}\left(\frac{\Delta}{\sigma}\right)^{3}
$$

and that this maximum occurs for a line ratio of $3+\sqrt{3}: 3-\sqrt{3}$. The limiting bin size for $f_{1,2}$ is written as

$$
\frac{\Delta_{2}}{F W H M}<2.2875 \lambda(R)^{1 / 3} N^{-1 / 6} \text {. }
$$

\subsection{Monte Carlo results}

In addition to the analytical approximations described above, we have performed Monte Carlo calculations as outlined in Sect. A.4. For our approximations of order 0,1 , and 2, corresponding to accounting for the number of photons only, both the number of photons and centroid and the number of photons, centroid, and dispersion, respectively, we found the following approximations:

$$
\frac{\Delta}{F W H M}=\min (1, y)
$$

with

order $0: y=\frac{0.5707}{x^{1 / 2}}\left(1+\frac{1.0}{x}\right)$,

order $1: y=\frac{1.404}{x^{1 / 4}}\left(1+\frac{18}{x}\right)$,

order $2: y=\frac{1.569}{x^{1 / 6}}\left(1+\frac{1.14}{x^{1 / 3}}\right)$,

where

order $0: x=N_{\mathrm{r}}(1+0.3 \ln R)$,

order $1: x=N_{\mathrm{r}}(1+0.1 \ln R)$,

order $2: x=N_{\mathrm{r}}(1+0.6 \ln R)$.

Here $N_{\mathrm{r}}$ is the number of photons within a resolution element and $R$ the number of resolution elements. The precise upper cutoff value of 1.0 is a little arbitrary, but we adopted it here for simplicity as the same as for our approximation of the data grid binning (see Sect. 5).

We show these approximations in Fig. 1. The asymptotic behaviour is as described by (8), (12), and (16), i.e. proportional to $N_{\mathrm{r}}^{-1 / 2}, N_{\mathrm{r}}^{-1 / 4}$, and $N_{\mathrm{r}}^{-1 / 6}$, respectively, although the normalisations for the Monte Carlo results as compared to the analytical approximations are higher by factors of $2.27,1.63$ and 1.38 , respectively. The difference is caused by the fact that the analytical approximation assumed that the maximum of the KolmogorovSmirnov statistic $D^{\prime}$ is reached at the $x$-value $x_{\mathrm{m}}$ where the cumulative distributions $F_{0}$ and $F_{1}$ reach their maximum distance.
In practice, because of statistical fluctuations the maximum for $D^{\prime}$ can also be reached for other values of $x$ close to $x_{\mathrm{m}}$, and this causes a somewhat more relaxed constraint on the bin size for the Monte Carlo results.

\subsection{Which approximation do we choose?}

We now compare the different approximations $f_{0}, f_{1}$, and $f_{2}$ as derived in the previous subsection. Figure 1 shows that the approximation $f_{1}$ yields an order of magnitude or more improvement over the classical approximation $f_{0}$. However, the approximation $f_{2}$ is only slightly better than $f_{1}$. Moreover, the computational burden of approximation $f_{2}$ is large. The evaluation of (14) is rather straightforward, although care should be taken with single machine precision; first the average energy $E_{\mathrm{a}}$ should be determined and then this value should be used in the estimation of $\tau$. A more serious problem is that the width of the lsf should be adjusted from $\sigma$ to $\sqrt{\sigma^{2}+\tau^{2}}$. If the lsf is a pure Gaussian, this can be carried out analytically; however, for a slightly non-Gaussian lsf the true lsf should be convolved in general numerically with a Gaussian of width $\tau$ to obtain the effective lsf for the particular bin, and the computational burden is very heavy. On the other hand, for $f_{1}$ only a shift in the lsf is sufficient.

Therefore we recommend using the linear approximation $f_{1}$. The optimal bin size is thus given by (17), (19), and (22).

\subsection{The effective area}

Above we showed how the optimal model energy grid can be determined, taking into account the possible presence of narrow spectral features, the number of resolution elements, and the flux of the source. We also need to account for the energy dependence of the effective area, however. In the previous section, we considered merely the effect of the spectral redistribution ( $\mathrm{rmf}$ ); here we consider the effective area (arf).

If the effective area $A_{j}(E)$ would be a constant $A_{j}$ within a model bin $j$, then for a photon flux $F_{j}$ in the bin the total count rate produced by this bin would be simply $A_{j} F_{j}$. This approach is actually used in the classical way of analysing spectra. In general $A_{j}(E)$ is not constant, however, and the above approach is justified only when all photons of the model bin have the energy of the bin centre. It is better to take into account not only the flux $F_{j}$ but also the average energy $E_{\mathrm{a}}$ of the photons within the model bin. This average energy $E_{\mathrm{a}}$ is in general not equal to the bin centre $E_{j}$, and hence we need to evaluate the effective area not at $E_{j}$ but at $E_{\mathrm{a}}$.

We consider here the most natural first-order extension, namely the assumption that the effective area within a model bin is a linear function of the energy. For each model bin $j$, we develop the effective area $A(E)$ as a Taylor series in $E-E_{j}$, which is written as

$A_{j}(E)=A\left(E_{j}\right)+A^{\prime}\left(E_{j}\right)\left(E-E_{j}\right)+\ldots$

The maximum relative deviation $\epsilon_{\max }$ from this approximation occurs when $E$ is at one of the bin boundaries. It is given by

$\epsilon_{\max }=\frac{1}{8}(\Delta E)^{2} A^{\prime \prime}\left(E_{j}\right) / A\left(E_{j}\right)$,

where $\Delta E$ is the model bin width. Therefore by using the approximation (24) we make at most a relative error in the effective area given by (25). This can be translated directly into an error in the predicted count rate by multiplying $\epsilon_{\max }$ by the photon flux $F_{j}$. 
The relative error in the count rate is thus also given by $\epsilon_{\max }$, which should be sufficiently small compared to the Poissonian fluctuations $1 / \sqrt{N_{\mathrm{r}}}$ in the relevant range.

We can do this in a more formal way by finding for which value of $\epsilon$ a test of the hypothesis that $N_{\mathrm{r}}$ is drawn from a Poissonian distribution with average value $\mu$ is effectively indistinguishable from tests that $N_{\mathrm{r}}$ is drawn from a distribution with mean $\mu(1+\epsilon)$. We have outlined such a procedure in Sect. A, and in the limit of large $\mu$ we write

$\mu+q_{\alpha} \sqrt{\mu}=\mu(1+\epsilon)+q_{k \alpha} \sqrt{\mu(1+\epsilon)}$,

where $\alpha$ is the size of the test and $q_{\alpha}$ is given by $G\left(q_{\alpha}\right)=1-\alpha$ with $G$ the cumulative normal probability distribution. Further $k \alpha$ is the size of the test when we use the approximation (24). In the limit of small $\epsilon$, the solution of (26) is given by

$\epsilon \simeq p(\alpha, k) / \sqrt{\left(N_{\mathrm{r}}\right)}$,

where we have approximated $\mu$ by the observed value $N_{\mathrm{r}}$, and where $p(\alpha, k)=q_{\alpha}-q_{k \alpha}$. For $\alpha=0.025$ and $k=2$, we obtain $p(\alpha, k)=0.31511$. Combining these results with (25), we obtain an expression for the model bin size

$\frac{\Delta}{F W H M}=\left(\frac{8 A p(\alpha, k)}{E_{j}^{2} A^{\prime \prime}}\right)^{0.5}\left(\frac{E_{j}}{F W H M}\right) N_{\mathrm{r}}^{-0.25}$.

The bin width constraint derived here depends upon the dimensionless curvature of the effective area $A / E_{j}^{2} A^{\prime \prime}$. In most parts of the energy range this is a number of order unity or less. Since the second prefactor $E_{j} / \mathrm{FWHM}$ is by definition the resolution of the instrument, we see by comparing (28) with (12) that, in general, (12) gives the most severe constraint upon the bin width. This is the case unless either the resolution becomes of order unity, which happens, for example for the Rosat (Truemper 1982) PSPC (Pfeffermann et al. 1987) detector at low energies, or the effective area curvature becomes large, which may happen, for example near the exponential cut-offs caused by filters.

Large effective area curvature due to the presence of exponential cut-offs is usually not a problem, since these cut-offs also cause the count rate to be low and hence weaken the binning requirements. Of course, discrete edges in the effective area should always be avoided in the sense that edges should always coincide with bin boundaries.

In practice, it is a little complicated to estimate from, for example a look-up table of the effective area its curvature, although this is not impossible. As a simplification for order of magnitude estimates, we use the case where $A(E)=A_{0} \mathrm{e}^{b E}$ locally, which after differentiation yields

$\sqrt{\frac{8 A}{E_{j}^{2} A^{\prime \prime}}}=\sqrt{8} \frac{\mathrm{d} \ln E}{\mathrm{~d} \ln A}$.

Inserting this into (28), we obtain our recommended bin size, as far as effective area curvature is concerned

$\frac{\Delta}{F W H M}=1.5877\left(\frac{\mathrm{d} \ln E}{\mathrm{~d} \ln A}\right)\left(\frac{E_{j}}{F W H M}\right) N_{\mathrm{r}}^{-0.25}$.

\subsection{Final remarks}

In the previous two subsections we have given the constraints for determining the optimal model energy grid. Combining both requirements (19) and (30) we obtain the following optimal bin size:

$\frac{\Delta}{F W H M}=\frac{1}{1 / w_{1}+1 / w_{\mathrm{a}}}$

where $w_{1}$ and $w_{\mathrm{a}}$ are the values of $\triangle / F W H M$ as calculated using (19) and (30), respectively.

This choice of model binning ensures that no significant errors are made either due to inaccuracies in the model or the effective area for flux distributions within the model bins that have $E_{\mathrm{a}} \neq E_{j}$.

\section{Data binning}

\subsection{Introduction}

Most X-ray detectors count the individual photons and do not register the exact energy value but a digitised version of it. Then a histogram is produced containing the number of events as a function of the energy. The bin size of these data channels ideally should not exceed the resolution of the instrument, otherwise important information may be lost. On the other hand, if the bin size is too small, one may have to deal with low statistics per data channel, insufficient sensitivity of statistical tests or a large computational overhead caused by the large number of data channels. Low numbers of counts per data channel can be alleviated by using $\mathrm{C}$-statistics (often slightly modified from the original definition by Cash 1979); computational overhead can be a burden for complex models, but insufficient sensitivity of statistical tests can lead to inefficient use of the information that is contained in a spectrum. We illustrate this inefficient use of information in Appendix C. In this section we derive the optimal bin size for the data channels.

\subsection{The Shannon theorem}

The Shannon (1949) sampling theorem states the following: let $f(x)$ be a continuous signal. Let $g(\omega)$ be its Fourier transform, given by

$g(\omega)=\int_{-\infty}^{\infty} f(x) \mathrm{e}^{\mathrm{i} \omega x} \mathrm{~d} x$.

If $g(\omega)=0$ for all $|\omega|>W$ for a given frequency $W$, then $f(x)$ is band limited, and in that case Shannon has shown that

$f(x)=f_{\mathrm{s}}(x) \equiv \sum_{n=-\infty}^{\infty} f(n \Delta) \frac{\sin \pi(x / \Delta-n)}{\pi(x / \Delta-n)}$.

In (33), the bin size $\Delta=1 / 2 W$. Thus, a band-limited signal is completely determined by its values at an equally spaced grid with spacing $\Delta$.

The above is used for continuous signals sampled at a discrete set of intervals $x_{i}$. However, X-ray spectra are essentially a histogram of the number of events as a function of channel number. We do not measure the signal at the data channel boundaries, but we measure the sum (integral) of the signal between the data channel boundaries. Hence for X-ray spectra it is more appropriate to study the integral of $f(x)$ instead of $f(x)$ itself.

We scale $f(x)$ to represent a true probability distribution. The cumulative probability density distribution function $F(x)$ is written as

$F(x)=\int_{-\infty}^{x} f(y) \mathrm{d} y$ 


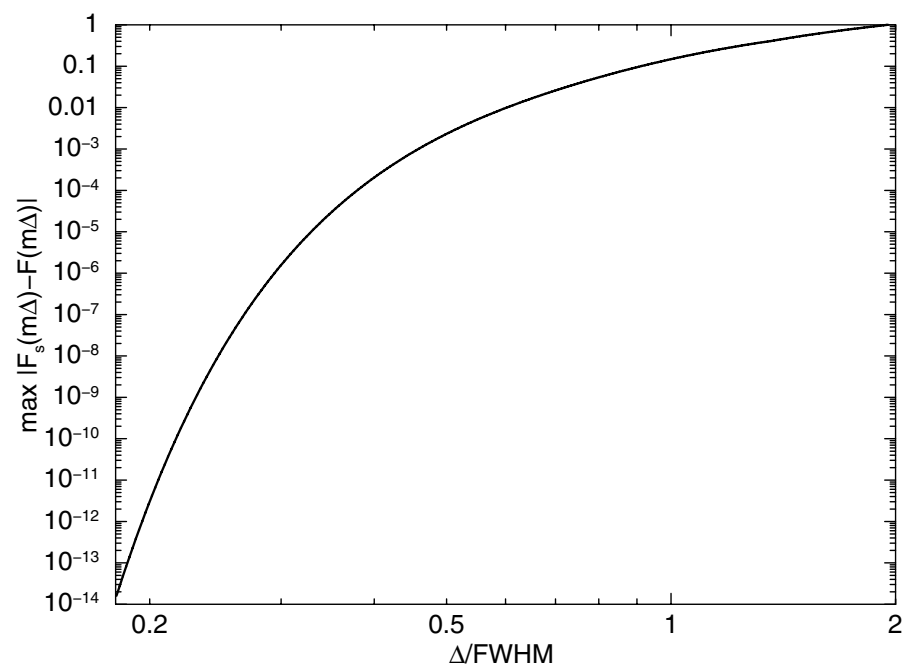

Fig. 2. Maximum difference for the cumulative distribution function of a Gaussian and its Shannon approximation, as a function of the bin size $\Delta$. The maximum is also taken over all phases of the energy grid with respect to the centre of the Gaussian.

If we insert the Shannon reconstruction (33) in (34), after interchanging the integration and summation and keeping into mind that we cannot evaluate $F(x)$ at all arbitrary points but only at those grid points $m \Delta$ for integer $m$ where also $f_{\mathrm{s}}$ is sampled, we obtain

$F_{\mathrm{s}}(m \Delta)=\frac{\Delta}{\pi} \sum_{n=-\infty}^{\infty} f(n \Delta)\left\{\frac{\pi}{2}+\operatorname{Si}[\pi(m-n)]\right\}$.

The function $\operatorname{Si}(x)$ is the sine-integral as defined, for example in Abramowitz \& Stegun (1965). The expression (35) for $F_{\mathrm{s}}$ equals $F$ if $f(x)$ is band limited. In that case at the grid points $F$ is completely determined by the value of $f$ at the grid points. By inverting this relation, one could express $f$ at the grid points as a unique linear combination of the $F$-values at the grid. Since Shannon's theorem states that $f(x)$ for arbitrary $x$ is determined completely by the $f$-values at the grid, we infer that $f(x)$ can be completely reconstructed from the discrete set of $F$-values. And then, by integrating this reconstructed $f(x), F(x)$ is also determined.

We conclude that $F(x)$ is also completely determined by the set of discrete values $F(m \Delta)$ at $x=m \Delta$ for integer values of $m$, provided that $f(x)$ is band limited.

For non-band-limited responses, we use (35) to approximate the true cumulative distribution function at the energy grid. In doing this, a small error is made. The errors can be calculated easily by comparing $F_{\mathrm{s}}(m \Delta)$ with the true $F(m \Delta)$ values. The binning $\Delta$ is sufficient if the sampling errors are sufficiently small compared with the Poissonian noise. We elaborate on what is sufficient below.

\subsection{Optimal binning of data}

We apply the theory outlined above to the case of a Gaussian redistribution function. We first determine the maximum difference $\delta_{k}$ between the cumulative Gaussian distribution function $F_{0}(x)$ and its Shannon approximation $F_{1}(x)=F_{\mathrm{s}}(x)$, as defined by (A.3). We show this quantity in Fig. 2 as a function of the bin size $\Delta$. It is seen that the Shannon approximation converges quickly to the true distribution for decreasing values $\Delta$. Using $\delta_{k}=\lambda_{k} / \sqrt{N}$ (see (A.9)), for any given value of $N$ and $\lambda_{k}=0.122$

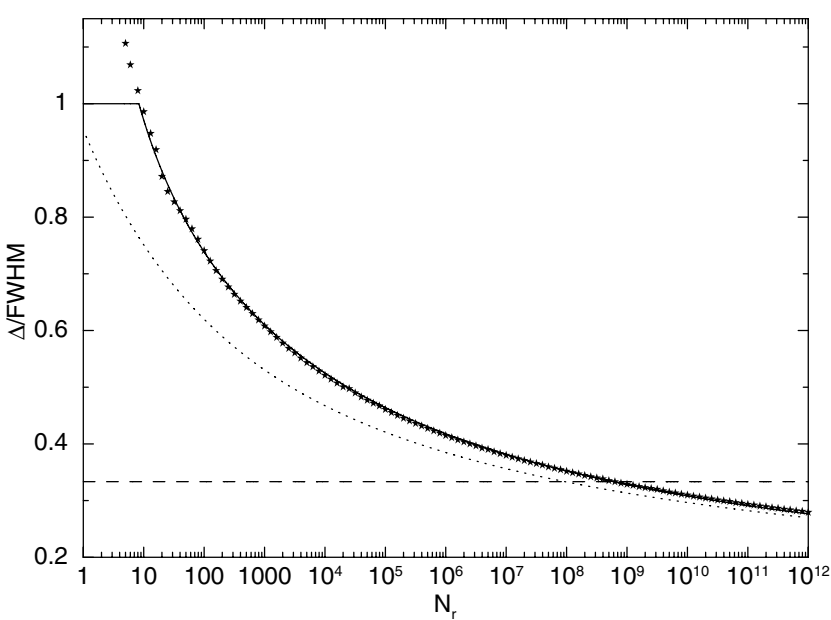

Fig. 3. Optimal bin size $\Delta$ for data binning with a Gaussian 1sf as a function of the number of counts per resolution element $N_{\mathrm{r}}$. Dotted curve: analytical approximation using Sect. A.3; stars: results of Monte Carlo calculation; solid line: our finally adopted bin size Eq. (36) based on a fit to our Monte Carlo results for large values of $N_{\mathrm{r}}$; dashed line: commonly adopted bin size 1/3 FWHM.

we can invert the relation and find the optimal bin size $\Delta$. This is plotted in Fig. 3. We have also verified this with the Monte Carlo method described before.

It is seen that the Monte Carlo results indeed converge to the analytical solution for large $N_{\mathrm{r}}$. For $N_{\mathrm{r}}=10$, the difference is $20 \%$. We have made a simple approximation to our Monte Carlo results (shown as the solid line in Fig. 3), where we cut it off to a constant value for $N_{\mathrm{r}}$ of less than about 2 . Both the Monte Carlo results and the analytical approximation break down at this small number of counts. In fact, for a small number of counts binning to about 1 FWHM is sufficient.

We also determined the dependence on the number of resolution elements $R$ for values of $R$ of $1,10,100,1000$, and 10000 . In general, the results even for $R=10^{4}$ are not too different from the $R=1$ case. We found a very simple approximation for the dependence on $R$ that is well described by the following function:

$\frac{\Delta}{F W H M}= \begin{cases}1 & \text { if } x \leq 2.119 ; \\ \frac{0.08+7.0 / x+1.8 / x^{2}}{1+5.9 / x} & \text { if } x>2.119,\end{cases}$

with

$x \equiv \ln \left[N_{\mathrm{r}}(1+0.20 \ln R)\right]$.

We note that for digitisation of analogue electronic signals that represent spectral information, a binning (=channel) criterion of 1/3 FWHM or better is often adopted (e.g. Davelaar 1979), since one does not know a priori the level of significance of the measured signal (see the dashed line in Fig. 3).

\subsection{Final remarks}

We have estimated conservative upper bounds for the required data bin size. In the case of multiple resolution elements, we have determined the bounds for the worst case phase of the grid with respect to the data. In practice, it is not likely that all resolution elements would have the worst possible alignment. However, we recommend using the conservative upper limits as given by (37). 


\section{J. S. Kaastra and J. A. M. Bleeker: Optimal binning}

Another issue is the determination of $N_{\mathrm{r}}$, the number of events within the resolution element. We have argued that an upper limit to $N_{\mathrm{r}}$ can be obtained by counting the number of events within one FWHM and multiplying it by the ratio of the total area under the lsf (should be equal to 1) to the area under the lsf within one FWHM (should be smaller than 1). For the Gaussian lsf, this ratio equals 1.314; for other lsfs, it is better to determine the ratio numerically and not to use the Gaussian value 1.314.

For the Gaussian lsf, the resolution depends only weakly upon the number of counts $N_{\mathrm{r}}$ (see Fig. 3). For low count rate parts of the spectrum, the binning rule of $1 / 3$ FWHM usually is too conservative.

Finally, in some cases the lsf may contain more than one component. For example, the grating spectra have higher order contributions. Other instruments have escape peaks or fluorescent contributions. In general it is advisable to determine the bin size for each of these components individually and simply take the smallest bin size as the final one.

\section{A practical example}

In order to demonstrate the benefits of the proposed binning schemes, We have applied them to the $85 \mathrm{ks}$ Chandra LETGS observation of Capella mentioned earlier. The spectrum spans the 0.86-175.55 $\AA$ range with a spectral resolution (FWHM) ranging between $0.040-0.076 \AA$. In Table 1 we show the number of data bins, model bins, and response matrix elements for different rebinning schemes of this spectrum. For simplicity we consider only the positive and negative first-order spectrum, and that the non-zero elements of the response matrix span a full range of four times the instrumental FWHM. We apply the algorithms as described in this paper to generate the data and model energy grids.

The highest resolution of $0.040 \AA$ occurs at short wavelengths, and the strongest line is the Fe XVII blend at 17.06 and $17.10 \AA$, with a maximum number of counts $N_{\mathrm{r}}$ of 15000 counts.

The first case we consider (case A afterwards) is that we adopt a data and model grid with constant step size for the full range. Accounting for the maximum $N_{\mathrm{r}}$ value, we have a data bin size of $0.02 \AA$ and a model bin size of $0.14 \mathrm{~m} \AA$.

Case B is similar to case A except that we account for the variable resolution of the instrument. This only decreases the number of bins by a factor of 1.375 .

In case $\mathrm{C}$ we account for the number of counts in each resolution element, but we still keep the "classical" approach of putting all photons at the centre of the model bins. The number of resolution elements $R$ in the spectrum is 3110 .

In case $\mathrm{D}$ we account for the average energy of the photons within a bin. The number of model bins and response elements needed drops by more than an order of magnitude for this case, as compared to case $\mathrm{C}$.

\section{The response matrix}

We have shown in the previous sections how the optimal model and data energy grids can be constructed. We have proposed that for the evaluation of the model spectrum both the number of photons in each bin as well as their average energy should be determined. We now determine how this impacts the concept of the response matrix.

In order to acquire high accuracy, we need to convolve the model spectrum for the bin, approximated as a $\delta$-function centred around $E_{\mathrm{a}}$, with the instrument response. In most cases we
Table 1. Number of data bins, model bins, and response matrix elements for different rebinning schemes of the Chandra LETGS spectrum of Capella.

\begin{tabular}{lccc}
\hline \hline Binning & Data bins & Model bins & $\begin{array}{c}\text { Response } \\
\text { elements }\end{array}$ \\
\hline A & $8.52 \times 10^{3}$ & $1.26 \times 10^{6}$ & $1.01 \times 10^{7}$ \\
B & $6.20 \times 10^{3}$ & $9.14 \times 10^{5}$ & $7.31 \times 10^{6}$ \\
C & $5.12 \times 10^{3}$ & $1.23 \times 10^{5}$ & $9.84 \times 10^{5}$ \\
D & $5.12 \times 10^{3}$ & $8.21 \times 10^{3}$ & $6.57 \times 10^{4}$ \\
\hline
\end{tabular}

cannot do this convolution analytically, so we have to make approximations. From our expressions for the observed count spectrum $s\left(E^{\prime}\right)$, Eqs. (1) and (2), it can be easily derived that the number of counts or count rate for data channel $i$ is given by

$S_{i}=\int_{E_{i 1}^{\prime}}^{E_{i 2}^{\prime}} \mathrm{d} E^{\prime} \int_{0}^{\infty} R\left(E^{\prime}, E\right) f(E) \mathrm{d} E$,

where, as before, $E_{i 1}^{\prime}$ and $E_{i 2}^{\prime}$ are the formal channel limits for data channel $i$ and $S_{i}$ is the observed count rate in counts/s for data channel $i$. Interchanging the order of the integrations and defining the mono-energetic response for data channel $i$ by $\tilde{R}_{i}(E)$ as follows:

$\tilde{R}_{i}(E) \equiv \int_{E_{i 1}^{\prime}}^{E_{i 2}^{\prime}} R\left(E^{\prime}, E\right) \mathrm{d} E^{\prime}$,

we obtain

$S_{i}=\int_{0}^{\infty} f(E) \tilde{R}_{i}(E) \mathrm{d} E$.

From the above equation we see that as long as we are interested in the observed count rate $S_{i}$ of a given data channel $i$, we get that number by integrating the model spectrum multiplied by the effective area $\tilde{R}_{i}(E)$ for that particular data channel. We have approximated $f(E)$ for each model bin $j$ by (9), so that (40) becomes

$S_{i}=\sum_{j} F_{j} \tilde{R}_{i}\left(E_{\mathrm{a}, j}\right)$

where as before $E_{\mathrm{a}, j}$ is the average energy of the photons in bin $j$ given by (10), and $F_{j}$ is the total photon flux for bin $j$, in e.g. photons $\mathrm{m}^{-2} \mathrm{~s}^{-1}$. It is seen from (41) that we need to evaluate $\tilde{R}_{i}$ not at the bin centre $E_{j}$ but at $E_{\mathrm{a}, j}$, as expected.

Formally we may split up $\tilde{R}_{i}(E)$ in an effective area part $A(E)$ and a redistribution part $\tilde{r}_{i}(E)$ in such a way that

$\tilde{R}_{i}(E)=A(E) \tilde{r}_{i}(E)$.

We have chosen our binning already in such a way that we have sufficient accuracy when the total effective area $A(E)$ within each model energy grid bin $j$ is approximated by a linear function of the photon energy $E$. Hence the arf-part of $\tilde{R}_{i}$ is of no concern. We only need to check how the redistribution (rmf) part $\tilde{r}_{i}$ can be calculated with sufficiently accuracy.

For $\tilde{r}_{i}$ the arguments are exactly the same as for $A(E)$ in the sense that if we approximate it locally for each bin $j$ by a linear 
function of energy, the maximum error that we make is proportional to the second derivative with respect to $E$ of $\tilde{r}_{i}$, cf. (25).

In fact, for a Gaussian redistribution function the following is straightforward to prove:

Theorem 1. Assume that for a given model energy bin j all photons are located at the upper bin boundary $E_{j}+\Delta / 2$. Suppose that for all data channels we approximate $\tilde{r}_{i}$ by a linear function of $E$, and the coefficients are the first two terms in the Taylor expansion around the bin centre $E_{j}$. Then the maximum error $\delta$ made in the cumulative count distribution (as a function of the data channel) is given by (12) in the limit of small $\Delta$.

The importance of the above theorem is that it shows that the binning for the model energy grid that we have chosen in Sect. 4 is also sufficiently accurate so that $\tilde{r}_{i}(E)$ can be approximated by a linear function of energy within a model energy bin $j$, for each data channel $i$. Since we already showed that our binning is also sufficient for a similar linear approximation to $A(E)$, it also follows that the total response $\tilde{R}_{i}(E)$ can be approximated by a linear function. Hence, within the bin $j$ we use

$\tilde{R}_{i}\left(E_{\mathrm{a}, j}\right)=\tilde{R}_{i}\left(E_{j}\right)+\frac{\mathrm{d} \tilde{R}_{i}}{\mathrm{~d} E_{j}}\left(E_{j}\right)\left(E_{\mathrm{a}, j}-E_{j}\right)$.

Inserting the above in (41) and comparing with (2) for the classical response matrix, we finally obtain

$S_{i}=\sum_{j} R_{i j} F_{j}+R_{i j}^{\prime}\left(E_{\mathrm{a}, j}-E_{j}\right) F_{j}$,

where $R_{i j}$ is the classical response matrix, evaluated for photons at the bin centre, and $R_{i j}^{\prime}$ is its derivative with respect to the photon energy $E_{j}$. In addition to the classical convolution, we thus get a second term containing the relative offset of the photons with respect to the bin centre. This is exactly what we intended to have when we argued that the number of bins could be reduced considerably by just taking that offset into account. It is just at the expense of an additional derivative matrix, which means only a factor of two more storage space and computation time. But for this extra expenditure we gained much more storage space and computational efficiency because the number of model bins is reduced by a factor between $10-100$.

Finally we make a practical note. The derivative $R_{i j}^{\prime}$ can be calculated in practice either analytically or by numerical differentiation. In the last case, it is more accurate to evaluate the derivative by taking the difference at $E_{j}+\Delta / 2$ and $E_{j}-\Delta / 2$, and, wherever possible, not to evaluate it at one of these boundaries and the bin centre. This last situation is perhaps only unavoidable at the first and last energy value.

Also, negative response values should be avoided. Thus it should be ensured that $R_{i j}+R_{i j}^{\prime} h$ is non-negative everywhere for $-\Delta / 2 \leq h \leq \Delta / 2$. This can be translated into the constraint that $R_{i j}^{\prime}$ should be limited always to the following interval:

$-2 R_{i j} / \Delta E \leq R_{i j}^{\prime} \leq 2 R_{i j} / \Delta E$.

Whenever the calculated value of $R_{i j}^{\prime}$ should exceed the above limits, the limiting value should be inserted instead. This situation may happen, for example for a Gaussian redistribution for responses a few $\sigma$ away from the centre, where the response falls off exponentially. However, the response $R_{i j}$ is small for those energies anyway, so this limitation is not serious; this is only because we want to avoid negative predicted count rates.

\section{Constructing the response matrix}

In the previous section we outlined the basic structure of the response matrix. For an accurate description of the spectrum, we need both the response matrix and its derivative with respect to the photon energy. We build on this to construct general response matrices for more complex situations.

\subsection{Dividing the response into components}

Usually only the non-zero matrix elements of a response matrix are stored and used. This is done to save both storage space and computational time. The procedure as used in XSPEC (Arnaud 1996) and the older versions of SPEX (Kaastra et al. 1996) is that for each model energy bin $j$ the relevant column of the response matrix is subdivided into groups. A group is a contiguous piece of the column with non-zero response. These groups are stored in a specific order; starting from the lowest energy, all groups belonging to a single photon energy are stored before turning to the next higher photon energy.

This is not optimal neither in terms of storage space nor computational efficiency, as illustrated by the following example. For the XMM-Newton/RGS, the response consists of a narrow Gaussian-like core with a broad scattering component due to the gratings in addition. The FWHM of the scattering component is $\sim 10$ times broader than the core of the response. As a result, if the response is saved as a classical matrix, we end up with one response group per energy, namely the combined core and wings response because they overlap in the region of the Gaussian-like core. As a result, the response file becomes large. This is not necessary because the scattering contribution with its ten times larger width needs to be specified only on a model energy grid with ten times fewer bins, compared to the Gaussian-like core. Thus, by separating out the core and the scattering contribution, the total size of the response matrix can be reduced by about a factor of 10 . Of course, as a consequence each contribution needs to carry its own model energy grid with it.

Therefore we propose subdividing the response matrix into its constituent components. Then for each response component, the optimal model energy grid can be determined according to the methods described in Sect. 4, and this model energy grid for the component can be stored together with the response matrix part of that component. Furthermore, at any energy each component may have at most one response group. If there were more response groups, the component should be subdivided further.

In X-ray detectors other than the RGS detector the subdivision could be different. For example, with CCD detectors one could split up the response into for components: the main diagonal, the Si fluorescence line, the escape peak, and a broad component due to split events.

\subsection{Complex configurations}

In most cases an observer analyses the data of a single source with a single spectrum and response for a single instrument. However, more complicated situations may arise. Examples are:

1. A spatially extended source, such as a cluster of galaxies, with observed spectra extracted from different regions of the detector, but with the need to be analysed simultaneously due to the overlap in point-spread function from one region to the other.

2. For the RGS of XMM-Newton, the actual data space in the dispersion direction is actually two-dimensional: the position 
$z$ of a photon on the detector and its energy or pulse height $E^{\prime}$ measured with the CCD detector. Spatially extended X-ray sources are characterised by spectra that are a function of both the energy $E$ and off-axis angle $\phi$. The sky photon distribution as a function of $(\phi, E)$ is then mapped onto the $\left(z, E^{\prime}\right)$ plane. One may analyse such sources by defining appropriate regions in both planes and evaluating the correct (overlapping) responses.

3. One may also fit simultaneously several time-dependent spectra using the same response, for example data obtained during a stellar flare.

It is relatively easy to model all these situations (provided that the instrument is understood sufficiently, of course), as we show below.

\subsection{Sky sectors}

First, the relevant part of the sky is subdivided into sectors, each sector corresponding to a particular region of the source, for example a circular annulus centred around the core of a cluster or an arbitrarily shaped piece of a supernova remnant, etc.

A sector may also be a point-like region on the sky. For example if there is a bright point source superimposed upon the diffuse emission of the cluster, we can define two sectors: an extended sector for the cluster emission and a point-like sector for the point source. Both sectors might even overlap, as this example shows.

Another example is the two nearby components of the close binary $\alpha$ Cen observed with the XMM-Newton instruments with overlapping psfs of both components. In that case we would have two point-like sky sectors, each sector corresponding to one of the double star components.

The model spectrum for each sky sector may and is different in general. For example, in the case of an AGN superimposed upon a cluster of galaxies, one might model the spectrum of the point-like AGN sector with a power law and the spectrum from the surrounding cluster emission with a thermal plasma model.

\subsection{Detector regions}

The observed count rate spectra are extracted in practice in different regions of the detector. It is necessary to distinguish the (sky) sectors and (detector) regions clearly. A detector region for the XMM-Newton EPIC camera would be for example a rectangular box, spanning a certain number of pixels in the $x$ - and $y$-directions. It may also be a circular or annular extraction region centred around a particular pixel of the detector, or whatever spatial filter is desired.

The detector regions need not coincide with the sky sectors and their number should not be equal. A good example of this is again an AGN superimposed upon a cluster of galaxies. The sky sector corresponding to the AGN is simply a point, while, for a finite instrumental psf, its extraction region at the detector is for example a circular region centred around the pixel corresponding to the sky position of the AGN.

Also, one could observe the same source with a number of different instruments and analyse the data simultaneously. In this case one would have only one sky sector but more detector regions, namely one for each participating instrument.

\subsection{Consequences for the response}

In all cases mentioned above, with more than one sky sector or more than one detector region involved, the response contribution for each combination of sky sector - detector region must be generated. In the spectral analysis, the model photon spectrum is calculated for each sky sector, and all these model spectra are convolved with the relevant response contributions to predict the count spectra for all detector regions. Each response contribution for a sky sector - detector region combination itself may consist again of different response components, as outlined in the previous subsection.

Combining all this, the total response matrix then consists of a list of components, each component corresponding to a particular sky sector and detector region. For example, we assume that the RGS has two response contributions: one corresponding to the core of the lsf and the other to the scattering wings. We assume that this instrument observes a triple star where the instrument cannot resolve two of the three components. The total response for this configuration then consists of 12 components. These components include three sky sectors, assuming each star has its own characteristic spectrum, times two detector regions, including a spectrum extracted around the two unresolved stars and one around the other star, times two instrumental contributions (the lsf core and scattering wings).

\section{Proposed file formats}

In the previous sections it was shown how the optimal data and model binning can be determined, and the corresponding optimal way to create the instrumental response. Now we focus on the possible data formats for these files.

A widely used response matrix format is NASA's OGIP ${ }^{1}$ (Corcoran et al. 1995) FITS format (Wells et al. 1981). This is used, for example, as the data format for XSPEC. There are a few reasons that we propose not to adhere to the OGIP format in its present form here, as listed below:

1. The OGIP response file format, as it is currently defined, does not account for the possibility of response derivatives. As was shown in previous sections, these derivatives are needed for the optimal binning.

2. As was shown in this work, it is more efficient to do the grouping within the response matrix differently, splitting the matrix into components where each component may have its own energy grid. This is not possible within the present OGIP format.

The FITS format used by the SPEX package version 2 obeys all the constraints that we outlined in this paper (Kaastra et al. 1996). This format is described in detail in the manual of that package $^{2}$.

\section{Conclusion}

In this paper, we derived the optimal bin size for both binning $\mathrm{X}$-ray data as well as binning the model energy grids used to calculate predicted X-ray spectra.

\footnotetext{
1 See the OGIP manual by K.A. Arnaud and I.M. George at https: // heasarc.gsfc .nasa.gov/docs/heasarc/ofwg/docs/spectra/ ogip_92_007/ogip_92_007.html

2 See www.sron.nl/spex
} 
The optimal bin size for model energy grids, in the way it is usually used (i.e. putting all photons at the bin centres) requires a large number of model bins. We have shown here that the number of model bins can be reduced by an order of magnitude or more using a variable binning scheme where not only the number of photons but also their average energy within a bin are calculated. The basic equations are (19), (30), and (31).

The data binning depends mostly on the number of counts per resolution element, and to a lesser extent on the number of resolution elements. The commonly used prescription of binning to $1 / 3$ of the instrumental FWHM is a safe number to use for most practical cases, but in most cases a somewhat courser sampling can be used without losing any sensitivity to test spectral models. The recommended binning scheme is given by (36).

Correspondingly, the response matrix needs to be extended to two components: the "classical" part and its derivative with respect to photon energy (See Eq. (44)). With these combined improvements a strong reduction of data storage needs and computational time is reached, which is very useful for the analysis of high-resolution spectra with large numbers of bins and computational complex spectral models.

Finally, we have outlined a few simple methods to reduce the size of the response matrix by splitting it into constituent components with different spectral resolution.

All these improvements are fully available in the spectral fitting package SPEX.

Acknowledgements. SRON is supported financially by NWO, the Netherlands Organization for Scientific Research.

\section{References}

Abramowitz, M., \& Stegun, I. A. 1965, Handbook of mathematical functions with formulas, graphs, and mathematical tables (New York: Dover Publications)

Arnaud, K. A. 1996, in Astronomical Data Analysis Software and Systems V, eds. G. H. Jacoby \& J. Barnes, ASP Conf. Ser., 101, 17

Brinkman, A. C., Gunsing, C. J. T., Kaastra, J. S., et al. 2000, ApJ, 530, L111

Cash, W. 1979, ApJ, 228, 939

Corcoran, M. F., Angelini, L., George, I., et al. 1995, in Astronomical Data Analysis Software and Systems IV, eds. R. A. Shaw, H. E. Payne, \& J. J. E. Hayes, ASP Conf. Ser., 77, 219

Davelaar, J. 1979, Ph.D. Thesis, Leiden University, The Netherlands

Den Herder, J. W., Brinkman, A. C., Kahn, S. M., et al. 2001, A\&A, 365, L7

Freeman, P., Doe, S., \& Siemiginowska, A. 2001, in Astronomical Data Analysis, SPIE Conf. Ser., 4477, 76

Jansen, F., Lumb, D., Altieri, B., et al. 2001, A\&A, 365, L1

Kaastra, J. S. 1999, in Lecture Notes in Physics, X-Ray Spectroscopy in Astrophysics, eds. J. van Paradijs, \& J. A. M. Bleeker (Berlin: Springer Verlag), 520, 269

Kaastra, J. S., Ebrero, J., Arav, N., et al. 2014a, A\&A, 570, A73

Kaastra, J. S., Kriss, G. A., Cappi, M., et al. 2014b, Science, 345, 64

Kaastra, J. S., Mewe, R., \& Nieuwenhuijzen, H. 1996, in UV and X-ray Spectroscopy of Astrophysical and Laboratory Plasmas, eds. K. Yamashita, \& T. Watanabe, 411

Mernier, F., de Plaa, J., Lovisari, L., et al. 2015, A\&A, 575, A37

Mewe, R., Raassen, A. J. J., Drake, J. J., et al. 2001, A\&A, 368, 888

Nandra, K., Barret, D., Barcons, X., et al. 2013, ArXiv e-prints [arXiv: 1306.2307]

Ness, J.-U., Mewe, R., Schmitt, J. H. M. M., et al. 2001, A\&A, 367, 282

Pfeffermann, E., Briel, U. G., Hippmann, H., et al. 1987, in Soft X-ray optics and technology, SPIE Conf. Ser., 733, 519

Shannon, C. E. 1949, Proc. of the IRE, 37, 10

Tanaka, Y., Inoue, H., \& Holt, S. S. 1994, PASJ, 46, L37

Truemper, J. 1982, Adv. Space Res., 2, 241

Weisskopf, M. C., O’dell, S. L., \& van Speybroeck, L. P. 1996, in Multilayer and Grazing Incidence X-Ray/EUV Optics III, SPIE Conf. Ser., 2805, 2

Wells, D. C., Greisen, E. W., \& Harten, R. H. 1981, A\&AS, 44, 363

Welsh, B. Y., Vallerga, J. V., Jelinsky, P., et al. 1990, Opt. Engineering, 29, 752 


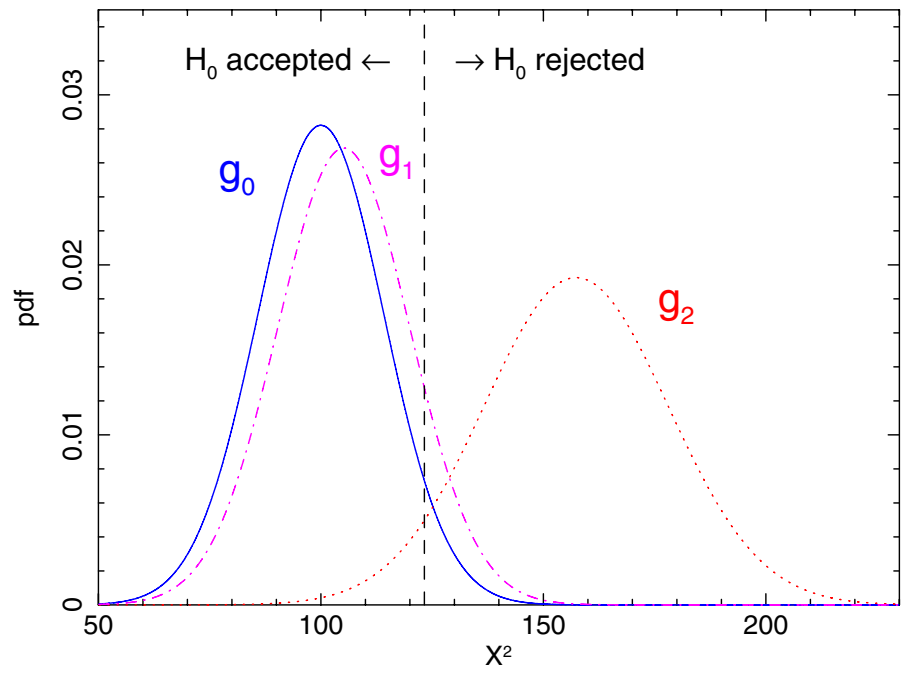

Fig. A.1. Probability distributions $g_{i}(T)$ for the example of $T=\chi^{2}$, corresponding to different spectral distribution functions $f_{0}$ (the "true" model underlying the data), $f_{1}$ (an approximation to $f_{0}$ based on finite sampling distance $\Delta$ ), and $f_{2}$ (an alternative physical model of the spectrum). The calculation is made for 100 degrees of freedom and $\alpha=\beta=0.025$. Thus, $2.5 \%$ of the area under $g_{0}$ is to the right of the dashed vertical line, and $2.5 \%$ of the area under $g_{2}$ is to the left of this same line. Further, $5 \%$ of the area under $g_{1}$ is to the right of the vertical line.

\section{Appendix A: Testing models}

\section{A.1. How is it possible to test an approximation to the spectrum?}

We make use of probability distributions to assess the accuracy of an approximation to the model spectrum.

Suppose that $f_{0}(x)$ is the unknown, true probability density function (pdf) describing the observed spectrum. Further let $f_{1}(x)$ be an approximation to $f_{0}$ (discretely sampled with a bin size $\Delta)$. Further let $f_{2}(x)$ be the pdf of an alternative spectral model. Ideally, one now designs a statistical test, based on some test statistics $T$. Choices for $T$ would be $\chi^{2}$ in a chi-squared test, or $D$ (see later) in a Kolmogorov-Smirnov test. The hypothesis $H_{0}$ that $f_{0}(x)$ describes the spectrum is accepted if $T$ is smaller than some threshold $c_{\alpha}$, and rejected if $T>c_{\alpha}$.

The probability $\alpha$ that $H_{0}$ will be rejected if it is true is called the size of the test. The probability that $H_{0}$ will be rejected if $H_{2}$ is true is called the power of the test and is denoted by $1-\beta$ as follows:

$\operatorname{Prob}\left(T>c_{\alpha} \mid H_{0}\right)=\alpha$,

$\operatorname{Prob}\left(T>c_{\alpha} \mid H_{2}\right)=1-\beta$.

An ideal statistical test of $H_{0}$ against $H_{2}$ has $\beta$ small (large power). However, in our case we are not interested in getting the most discriminative power between the alternatives $H_{0}$ and $H_{2}$, but we want to know how well the approximation $f_{1}$ works in reaching the right conclusions about the model. When we use $f_{1}$ instead of $f_{0}$, we want to reach the same conclusion in the majority of all cases. We define the size of the test of $H_{1}$ versus $\mathrm{H}_{2}$ to be $k \alpha$ with the same $\alpha$ as above. For $k=1$, both distributions would of course be equal, but this happens only for bin size $\Delta=0$.

We adopt as a working value $k=2, \alpha=0.025$. That is, using a classical $\chi^{2}$ test $H_{1}$ will be rejected against $H_{2}$ in $5 \%$ of the cases, the usual criterion in $\chi^{2}$-analysis. This means that in practice $H_{1}$ reaches the same correct conclusion as $H_{0}$ in $0.95 / 0.975 \simeq 0.975$ of all cases. For an example, see Fig. A.1.

\section{A.2. Statistical tests}

Up to now we have not specified the test statistic $T$. It is common to do a $\chi^{2}$-test. However, the $\chi^{2}$-test has some drawbacks, in particular for small sample sizes. It can be shown, for instance, for a Gaussian distribution that the test is rather insensitive in discriminating relatively large relative differences in the tails of the distribution.

For our purpose, a Kolmogorov-Smirnov test is more appropriate. This powerful, non-parametric test is based upon the test statistic $D$ given by

$D=\max \left|S_{N}(x)-F(x)\right|$,

where $S_{N}(x)$ is the observed cumulative distribution for the sample of size $N$, and $F(x)$ is the cumulative distribution for the model that is tested. Clearly, if $D$ is large, $F(x)$ is a bad representation of the observed data set. The statistic $D^{\prime} \equiv \sqrt{N} D$ for large $N$ (typically 10 or larger) has the limiting Kolmogorov-Smirnov distribution, with expectation value $\sqrt{\pi / 2} \ln 2=0.86873$ and standard deviation $\pi \sqrt{1 / 12-(\ln 2)^{2} / 2 \pi}=0.26033$. The hypothesis that the true cumulative distribution is $F$ will be rejected if $D^{\prime}>c_{\alpha}$, where the critical value $c_{\alpha}$ corresponds to a certain size $\alpha$ of the test.

A good criterion for determining the optimal bin size $\Delta$ is that the maximum difference

$\delta_{k} \equiv \max _{m}\left|F_{1}(m \Delta)-F_{0}(m \Delta)\right|$

should be sufficiently small compared to the statistical fluctuations described by $D^{\prime}$. Here $F_{0}(x)$ is the true underlying cumulative distribution and $F_{1}(x)$ is again its approximation, which we assume here are sampled on an energy grid with spacing $\Delta$.

We can apply exactly the same arguments as discussed before and use Fig. A.1 with $T=D^{\prime}$.

The Kolmogorov-Smirnov test was designed for comparing distributions based on drawing $N$ independent values from the distribution $f(x)$; it is assumed that $x$ can have any real value within the interval where $f(x)>0$. In our case, because of the binning with finite bin size $\Delta$, we first round the observed values of $x$ to a set of discrete values and then perform a test. This makes a significant difference; for a binned Gaussian redistribution function $f(x)$ with $N$ large, we found from numerical simulations that the average value of $D^{\prime}$ equals $0.64,0.69,0.77$, and 0.84 for $\Delta$ equal to $0.5,0.3,0.1$, and 0.01 , respectively. Thus, convergence to the asymptotic value for $D^{\prime}$ of 0.86873 for $\Delta \rightarrow 0$ is rather slow.

\section{A.3. Analytical approximation}

We now make a simple analytical approximation for the optimal bin size. We first determine the maximum deviation of the approximation $F_{1}$ from $F_{0}: \delta_{k}$ given by (A.3). This maximum occurs at a given value of $x_{\mathrm{m}}$. We are interested in tests of $H_{0}$ versus alternatives $\mathrm{H}_{2}$, and as we have seen (Fig. A.1), this requires knowledge of the distribution $g_{0}\left(D^{\prime}\right)$ at high values of $D^{\prime}$ (typically the highest $5 \%$ of the distribution). Because the observed sample is drawn from the distribution $f_{0}(x), D^{\prime}$ can be reached at any value of $x$, wherever the random fluctuations reach the highest values. However, in the test using $f_{1}$ as an approximation to $f_{0}$, the $x$-values near $x_{\mathrm{m}}$ have a higher probability of yielding 
the maximum because in addition to the random noise there is an offset $\lambda_{k}$ due to the maximum difference between $F_{0}$ and $F_{1}$. In fact, we may approximate the tail of the distributions $g_{0}$ and $g_{1}$ by

$g_{1}\left(x+\lambda_{k}\right) \simeq g_{0}(x)$.

We now approximate the distribution function $g_{0}$ of the $D^{\prime}$ statistic by the limiting Kolmogorov-Smirnov distribution $g_{\mathrm{KS}}$. We thus have to solve for

$G_{0}\left(c_{\alpha}\right)=1-\alpha$,

$G_{1}\left(c_{\alpha}\right)=1-k \alpha$,

which translates into

$G_{\mathrm{KS}}\left(c_{\alpha}\right)=1-\alpha$,

$G_{\mathrm{KS}}\left(c_{\alpha}-\lambda_{k}\right)=1-k \alpha$.

For our choice of $\alpha=0.025$ and $k=2$, we obtain $\lambda_{k}=0.122$. We note that the dependence on $\alpha$ is weak: for $\alpha=0.01$ or $\alpha=0.05$, we get $\lambda_{k}$ values of 0.110 and 0.134 , respectively. To get the optimal bin size for any value of $N$, we calculate

$\epsilon \equiv \lambda_{k} / \sqrt{N}$.

We then approximate $\delta_{k} \simeq \epsilon$ and using (A.3) we can determine for which bin size $\Delta$ this occurs.

In evaluating (A.3) for a given cumulative distribution $F_{0}(x)$ and $F_{1}(x)$, it is useful to remember that the extrema of $F_{1}(x)-$ $F_{0}(x)$ occur where its derivative is zero, i.e. where $f_{1}(x)=f_{0}(x)$, where $f_{0}$ and $f_{1}$ are the corresponding probability density distributions.

\section{A.4. Monte Carlo calculations}

The analytical approximations described above yield interesting asymptotic limits. However, we need to test their accuracy and applicability. To do this, we have used Monte Carlo simulations. For a set of sample sizes $N$ and bin sizes $\Delta$, we calculated the true distribution function $F_{0}$ and its approximation $F_{1}$. Using $F_{0}$, we have drawn a large number (typically $10^{7}$ ) of realisations. For each random realisation, we calculated $D^{\prime}$ against both models $F_{0}$ and $F_{1}$. Combining all runs, we determined the cumulative distributions $G_{0}\left(D^{\prime}\right)$ and $G_{1}\left(D^{\prime}\right)$. From these distributions, we determined the critical value $c_{\alpha}$ where $G_{0}\left(c_{\alpha}\right)=1-\alpha$ (using $\alpha=0.025$ ). For a given sample size $N$, we then varied $\Delta$ in such a way that $G_{1}\left(c_{\alpha}\right)=1-k \alpha$ with as before $k=2$. This procedure then yields the optimal bin size $\Delta$ as a function of $N$.

The Monte Carlo method works well for large values for $N$. Because we work with $\alpha=0.025$, for $N \leq 1 / \alpha$ the distribution $G_{0}(x)$ begins suffering from discontinuous steps due to the limited number $N$ of values that the observed distribution $S_{N}(x)$ can have. In practice, this is not a major problem as our results to be presented later show that in those cases the derived bin sizes become of the order of the FWHM of the instrumental response function anyway.

\section{A.5. Extension to complex spectra and instruments}

The estimates that we derived for determining bin sizes hold for any spectrum. The optimal bin size $\Delta$ as a function of $N$ can be determined using the analytical approximations or numerical calculations outlined above.

This method is not always practical, however. First, the spectral resolution can be a function of the energy, hence binning with a uniform bin width over the entire spectrum is not always desired.

Further, regions with poor statistics contain less information than regions with good statistics, hence can be sampled on a much coarser grid.

Finally, a spectrum often extends over a much wider energy band than the spectral resolution of the detector. In order to estimate $\epsilon$, this would require that first the model spectrum, convolved with the instrument response, should be used to determine $f_{0}(x)$. However, the true model spectrum is known in general only after spectral fitting. But the spectral fitting can be done only after a bin size $\Delta$ has been set.

To overcome these problems, we consider the instrumental line-spread function (lsf). This is the response (probability distribution) of mono-energetic photons that the instrument measures. Most X-ray spectrometers have a lsf with a characteristic width $\Delta E$ smaller than the incoming photon energy $E$, and we only consider instruments obeying that condition; there is little use in binning data from instruments lacking spectral resolution. To be more precise, we define a resolution element to be a region in data space corresponding to the FWHM of the detector; this FWHM is called $\Delta E$.

We now consider a region $r$ of the spectrum with a width of the order of $\Delta E$. In this region the observed spectrum is mainly determined by the convolution of the instrumental lsf with the true model spectrum in an energy band with a width of the order of $\Delta E$. We ignore here any substantial tails in the lsf, since they are of little importance in the determination of the optimal bin size/resolution.

If all $N_{\mathrm{r}}$ photons within the resolution element $r$ would be emitted at the same energy $E_{\mathrm{r}}$, and no photons would be emitted at any other energy, the observed spectrum would be simply the lsf for energy $E_{\mathrm{r}}$ multiplied by the number of photons $N_{\mathrm{r}}$. For this situation, we can easily evaluate $\lambda_{k}$ for a given binning.

If the spectrum is not mono-energetic, then the observed spectrum within the resolution element is the convolution of the full photon distribution with the lsf. In this case, the observed spectrum $S_{\mathrm{r}}$ in resolution element $r$ is a weighted sum of the photon spectra $F_{i}$ in a number of usually adjacent resolution elements $i$, say $S_{\mathrm{r}}=\sum w_{i} F_{i}$. Because for a set of weights $w_{i}$ in general $\sqrt{\sum w_{i}^{2}} \leq \sum w_{i}$, the squared modelling uncertainties $\epsilon_{\mathrm{r}}^{2}$ are smaller for the case of a broadband spectrum than for a mono-energetic spectrum. It follows that if we determine $\epsilon_{\mathrm{r}}^{2}$ for the mono-energetic case from the sampling errors in the lsf, this is an upper limit to the true value for $\epsilon^{2}$ within the resolution element $r$.

We now need to combine the resolution elements. Suppose that in each of the $R$ resolution elements we perform a Kolmogorov-Smirnov test. For the maximum of $D^{\prime}$ over all resolution elements $r$, we test

$\delta \equiv \max _{\mathrm{r}} \sqrt{N_{\mathrm{r}}} D_{\mathrm{r}}$

where as before $N_{\mathrm{r}}$ is the number of photons in the resolution element $r$, and $D_{\mathrm{r}}$ is given by (A.2) over the interval $r$. All the $\sqrt{N_{\mathrm{r}}} D_{\mathrm{r}}$ values are independently distributed, hence the cumulative distribution function for their maximum is simply the $R$ th power of a single stochastic variable with the relevant distribution. Therefore we obtain

$\left[G_{0}\left(c_{\alpha}\right)\right]^{R}=1-\alpha$,

$\left[G_{1}\left(c_{\alpha}\right)\right]^{R}=1-k \alpha$. 


\section{Appendix B: Determining the grids in practice}

Based upon the theory developed in the previous sections, we present here a practical set of algorithms for the determination of both the optimal data binning and the model energy grid determination. This may be helpful for the practical purpose of developing software for a particular instrument to construct the relevant response matrix.

\section{B.1. Creating the data bins}

Given an observed spectrum obtained by some instrument, the following steps should be performed to generate an optimally binned spectrum.

1. Determine for each original data channel $i$ the nominal energy $E_{j 0}$, defined as the energy for which the response at channel $i$ reaches its maximum value. In most cases, this is the nominal channel energy.

2. Determine for each data channel $i$ the limiting points $(i 1, i 2)$ for the FWHM in such a way that $R_{k, j 0} \geq 0.5 R_{i, j 0}$ for all $i 1 \leq k \leq i 2$, while the range of $(i 1, i 2)$ is as broad as possible.

3. By (linear) interpolation, determine for each data channel the points (fractional channel numbers) $c 1$ and $c 2$ near $i 1$ and $i 2$, where the response is actually half its maximum value. By virtue of the previous step, the absolute difference $|c 1-i 1|$ and $|c 2-i 2|$ never can exceed 1 .

4. Determine for each data channel $i$ the FWHM $c_{i}$ in units of channels, by calculating $c 2-c 1$. Assure that $c_{i}$ is at least 1 .

5. Determine for each original data channel $i$ the FWHM in energy units (e.g. in keV). Call this $W_{i}$. This and the previous steps may of course also be performed directly using instrument calibration data.

6. Determine the number of resolution elements $R$ by the following approximation:

$$
R=\sum_{i} \frac{1}{c_{i}}
$$

7. Determine for each bin the effective number of events $N_{\mathrm{r}}$ from the following expressions:

$$
\begin{aligned}
C_{\mathrm{r}} & =\sum_{k=i 1-1}^{i 2+1} C_{k}, \\
h_{\mathrm{r}} & =\sum_{k=1}^{N_{c}} R_{k, j 0} \mid \sum_{k=i 1-1}^{i 2+1} R_{k, j 0}, \\
N_{\mathrm{r}} & =C_{\mathrm{r}} h_{\mathrm{r}} .
\end{aligned}
$$

In the above, $C_{k}$ is the number of observed counts in channel $k$, and $N_{\mathrm{c}}$ is the total number of channels. Take care that in the summations $i 1-1$ and $i 2+1$ are not out of their valid range $\left(1, N_{\mathrm{c}}\right)$. If for some reason there is not a first-order approximation available for the response matrix $R_{k, j}$ then one might simply approximate $h_{\mathrm{r}}$ from e.g. the Gaussian approximation, namely $h_{\mathrm{r}}=1.314$; cf. Sect. 5.4. This is justified since the optimal bin size is not a strong function of $N_{\mathrm{r}}$; cf. Fig. 3. Even a factor of two error in $N_{\mathrm{r}}$ in most cases does not affect the optimal binning too much.

8. Using (36), determine for each data channel the optimal data bin size in terms of the FWHM. The true bin size $b_{i}$ in terms of number of data channels is obtained by multiplying this by $c_{i}$ calculated above during step 4 . Make $b_{i}$ an integer number by ignoring all decimals (rounding it to below), but take care that $b_{i}$ is at least 1 .

9. It is now time to merge the data channels into bins. In a loop over all data channels, start with the first data channel. Name the current channel $i$. Take in principle all channels $k$ from channel $i$ to $i+b_{i}-1$ together. However, check that the bin size does not decrease significantly over the rebinning range. In order to do that check, determine for all $k$ between $i$ and $i+b_{i}-1$ the minimum $a_{i}$ of $k+b_{k}$. Extend the summation only from channel $i$ to $a_{i}-1$. In the next step of the merging, $a_{i}$ becomes the new starting value $i$. The process is finished when $a_{i}$ exceeds $N_{c}$.

\section{B.2. Creating the model bins}

After having created the data bins, it is possible to generate the model energy bins. Some of the information obtained from the previous steps that created the data bins is needed.

The following steps need to be taken:

1. Sort the FWHM of the data bins in energy units $\left(W_{i}\right)$ as a function of the corresponding energies $E_{j 0}$. Use this array to interpolate any true FWHM later. Also use the corresponding values of $N_{\mathrm{r}}$ derived during that same stage. Alternatively, one may use directly the FWHM as obtained from calibration files.

2. Choose an appropriate start and end energy, e.g. the nominal lower and upper energy of the first and last data bin with an offset of a few FWHMs (for a Gaussian, about 3 FWHM is sufficient). In the case of a lsf with broad wings (like the scattering due to the RGS gratings), it may be necessary to take an even broader energy range.

3. In a loop over all energies, as determined in the previous steps, calculate the bin size in units of the FWHM using (19).

4. Also, determine the effective area factor $\frac{\mathrm{d} \ln E}{\mathrm{~d} \ln A}$ for each energy; one may do that using a linear approximation.

5. For the same energies, determine the necessary bin width in units of the FWHM using Eq. (31). Combining this with the FWHMs determined above gives for these energies the optimal model bin size $\Delta E$ in $\mathrm{keV}$.

6. Now the final energy grid can be created. Start at the lowest energy $E_{1,1}$, and interpolate in the $\Delta E$ table the appropriate $\Delta E\left(E_{1,1}\right)$ value for the current energy. The upper bin boundary $E_{2,1}$ of the first bin is then simply $E_{1,1}+\Delta E\left(E_{1,1}\right)$.

7. Using the recursive scheme $E_{1, j}=E_{2, j-1}, E_{2, j}=E_{1, j}+$ $\Delta E\left(E_{1, j}\right)$ determine all bin boundaries until the maximum energy has been reached. The bin centres are simply defined as $E_{j}=0.5\left(E_{1, j}+E_{2, j}\right)$.

8. Finally, if there are any sharp edges in the effective area of the instrument, it is necessary to add these edges to the list of bin boundaries. All edges should coincide with bin boundaries.

\section{Appendix C: Oversampling of data}

If a spectrum is binned with data bins that are too narrow, important information about the spectrum is lost. This was shown in a more formal way, for instance by Kaastra (1999, Sect. 2.6) using $\chi^{2}$-statistics. The argument also holds for lower count statistics. Here we give a practical example using C-statistics, but our conclusions are independent of the precise statistics used.

We have simulated a spectrum consisting of a $50 \mathrm{eV}$ blackbody plus a weak Gaussian emission line at $30 \AA$ with a FWHM of $1 \AA$. The instrument over-samples the spectrum with a bin size 


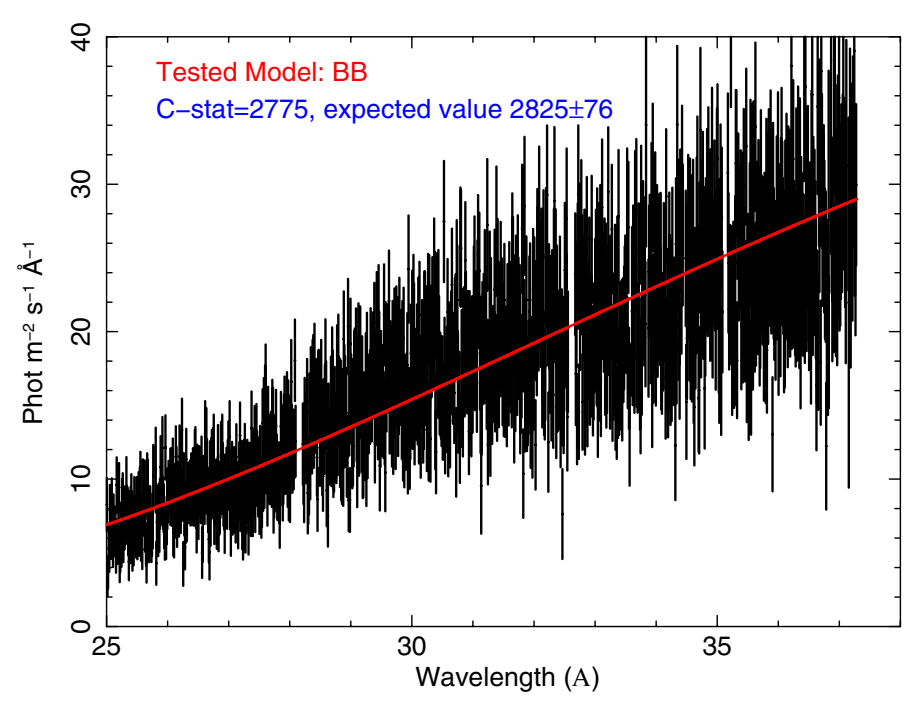

Fig. C.1. Simulated X-ray spectrum (data points) sampled with a bin size of $0.01 \AA$ and fitted with a blackbody with a temperature of $50 \mathrm{eV}$ (solid line). The goodness of the fit is indicated in the figure.

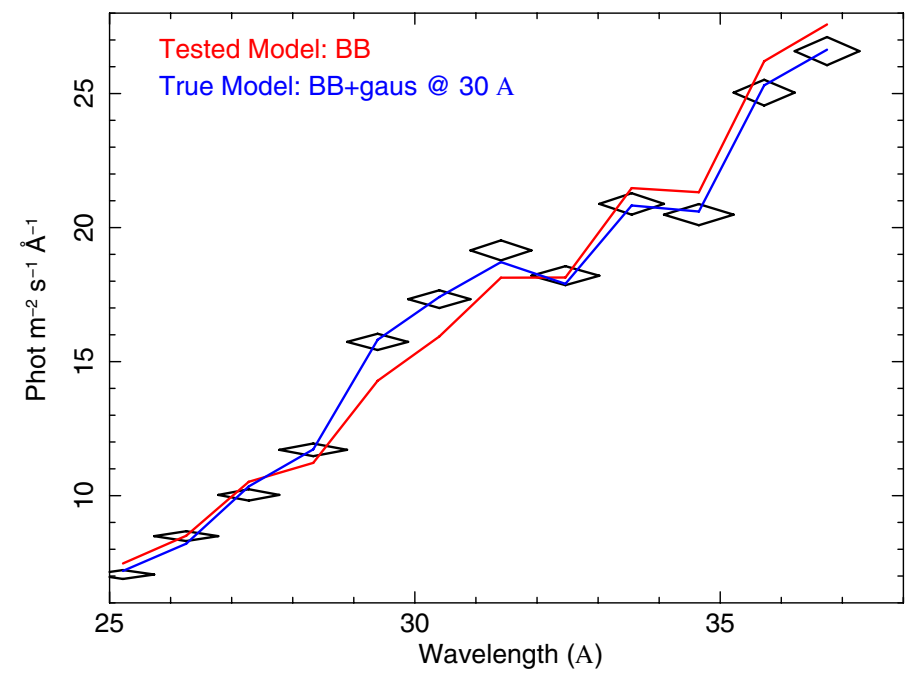

Fig. C.2. Same data as Fig. C.1 but rebinned by a factor of 100 (diamonds). The same best-fit model of Fig. C.1 using a simple blackbody with a temperature of $50 \mathrm{eV}$ is indicated with the red line. The blue line indicates the model for the source that entered the simulation: the sum of a blackbody spectrum and a weak Gaussian line at $30 \AA$.

of $0.01 \AA$. The spectrum was fit with a simple blackbody (without a Gaussian line) using C-statistics and the fit was "perfect", with a C-stat value of 2775 , to be compared to the expected value of $2825 \pm 76$ for the best-fit model. See Fig. C.1 for the fit. For the fitting we have used the SPEX software, which calculates for each fit the expected value of C-stat (in this case 2825) and its variance. This expected value is simple to calculated knowing the expected number of counts from the best-fit model and the observed number of counts from the data in each data channel.

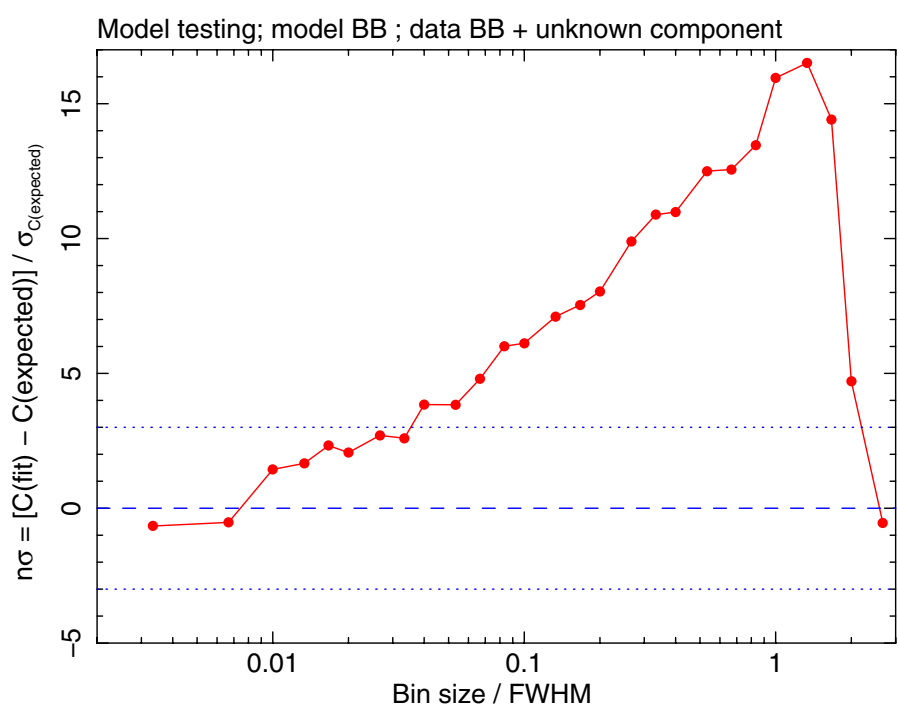

Fig. C.3. Best-fit C-statistic of the spectrum of Fig. C.1 when fitted with a simple blackbody. We subtract here the expected value of $\mathrm{C}$-stat and scale by its expected root-mean-square value, so that it is expressed in equivalent number of $\sigma$. The dotted lines indicate the $\pm 3 \sigma$ range; when the best fit is outside this $\pm 3 \sigma$ range, most investigators would reject the model (in this case a simple blackbody). The FWHM used in this figure is the FWHM of the additional Gaussian component in the spectrum (1 A).

Thus, with this bin size, the observer would conclude that a simple blackbody spectrum yields an accurate representation of the data, and there is no need to add more components because the present fit is already statistically acceptable. However, in Fig. C.2 we show the same data and model rebinned by a factor of 100 (i.e. $1 \AA$ wide bins). It is seen only at this bin size that the source has an emission line in addition to the simple blackbody model.

We elaborate on this by rebinning the original spectrum of Fig. C.1 by different factors, fitting the spectrum with a single blackbody component and comparing the best-fit $\mathrm{C}$-stat with the relevant expected $\mathrm{C}$-stat and its variance. The results are shown in Fig. C.3. It is clear that for all bin sizes less than about $0.04 \AA$ the observer cannot reject the simple blackbody hypothesis, and has no reason to add the Gaussian component to the model. Only for bin sizes of the order of $1 \AA$, the simple blackbody hypothesis can be rejected at the $>15 \sigma$ significance level. For higher binning factors, the bin size becomes larger than the width of the Gaussian line, and the significance drops again.

While the proper flux of the Gaussian line, including its uncertainties, is recovered at the proper value, regardless of the binning, when the additional Gaussian is introduced in the model, the detection and proof of existence of this component is only achieved when the spectrum is optimally binned, in this case (relatively weak line) with a bin size close to the FWHM of the Gaussian. 